\title{
Railway track component condition monitoring using optical fibre Bragg grating sensors
}

\author{
S. J. Buggy ${ }^{\mathrm{a} 1}$, S.W. James ${ }^{\mathrm{a}}$, S Staines ${ }^{\mathrm{a}}$, R. Carroll ${ }^{\mathrm{b}}$, P. Kitson ${ }^{\mathrm{c}}$, \\ D. Farrington ${ }^{c}$, L. Drewett ${ }^{c}, J^{J} J a i s w a l^{c}$ and R.P. Tatam ${ }^{a}$ \\ aDepartment of Engineering Photonics, School of Engineering, Cranfield University MK43 OAL, UK; \\ ${ }^{b}$ Stagecoach Supertram, Nunnery Depot, Woodbourn Road, Sheffield, S9 3LS, UK; \\ 'TATA Steel, Tata Steel RD\&T, Teesside Technology Centre, Grangetown, Middlesbrough, TS6 6US, UK.
}

\begin{abstract}
The use of optical fibre Bragg grating (FBG) strain sensors to monitor the condition of safety critical rail components is investigated. Fishplates, switchblades and stretcher bars on the Stagecoach Supertram tramway in Sheffield in the UK have been instrumented with arrays of FBG sensors. The dynamic strain signatures induced by the passage of a tram over the instrumented components have been analyzed to identify features indicative of changes in the condition of the components.
\end{abstract}

Keywords: fibre Bragg grating sensors, railway components, condition monitoring

\section{Introduction}

Ever-increasing passenger numbers and moves toward longer operating hours are placing considerable demands on rail transportation networks, generating a requirement to monitor and maintain the networks with minimal disruption to service. The condition of many aspects of railway infrastructure is still assessed by visual inspection by staff walking the line. There is increasing interest in the use of remote condition monitoring (RCM). Mainline networks employ instrumented trains that employ a range of sensors, ultrasonic systems, high-speed imaging and laser scanning devices to monitor track and overhead line condition, and there is increasing use of RCM instrumentation mounted on train fleets [1]. Track-side RCM can facilitate continuous monitoring of track condition, and is becoming a more viable option with the ability to communicate wirelessly with sensors and data loggers [2]. Distributed and multiplexed optical fibre sensors (OFSs) offer a number of attractive features for monitoring large- scale infrastructure due to the low-loss nature of the optical fibre, which acts as both the sensing medium and the transmission line, and offer particular benefits when making measurements in the presence of large electromagnetic fields. This sensing technology is gaining commercial traction in a number of industries, in particular oil and gas and renewable energy generation, and has proven capability for operating in harsh environments [3]. OFSs have been proposed for a wide scope of applications within the rail industry, including rail and wheel condition monitoring [4], train detection [5], weight and speed measurement [6], axle number counting $[7,8,9]$, rail deformation monitoring $[10,11]$, monitoring of pantograph-catenary interaction [12] and the monitoring of rail infrastructure, for example bridges [13] and tunnels.

In this paper the development and field-testing of a fibre Bragg grating (FBG) based sensing system that is to be used as a means of monitoring rail components at multiple locations on a tram network in order to identify a change from their normal operating condition is reported. Preliminary results were published previously in [14]. The rail sub-components that have been investigated are fishplated joints, insulated joints, switchblades and stretcher bars, the failure of which can lead to catastrophic failure [15].

\section{Applications of optical fibre Bragg grating sensors in the rail industry}

Optical fibre Bragg gratings (FBGs) are a well-established sensing technology that have achieved commercial success in a number of industrial sectors, but are still the subject of significant academic

${ }^{1}$ SJB's current address: Vestas Technology UK Ltd, West Medina Mills, Newport, PO30 5TR Isle of Wight, UK 
research. In essence, an FBG offers a means for labeling a short section of optical fibre (typically of length $5 \mathrm{~mm}$ ) to allow it to be interrogated to provide information on perturbations experienced by the fibre in response to the local environment. The basis of the technology is the measurement of changes in the wavelength of light reflected from the FBG in response to environmental perturbation. Arrays of sensors can be multiplexed within a single optical fibre, with arbitrary physical separation, allowing a high density of sensors to be deployed where large strain gradients are expected, or a sparse array to monitor widely spaced points. The sensor elements are often wavelength-division multiplexed, with each FBG in the array fabricated to reflect a distinct wavelength. There is a range of sensor interrogation approaches that allow the demodulation of the sensors at data rates of upto $100 \mathrm{~s} \mathrm{kHz}$, with pm wavelength resolution (corresponding to $\mu \mathrm{m} / \mathrm{m}$ strain resolution) $[16,17]$.

As mentioned in the introduction, the applications proposed in the rail sector span infrastructure, traffic movement, track components, catenary and the vehicles themselves. A brief review of previous reports is provided here.

The modal characteristics of the rail and sleepers have been investigated using OFSs with the aim of identifying signatures that indicate the presence of damage or defects $[18,19]$. FBGs bonded to rails have been used to monitor local deformation of the rail in response to the loading by a passing train and to monitor switch arms during transitions [10]. The displacement of a switch and the sleepers on which it was mounted were monitored on a high speed line, showing good correlation with daily temperature changes [20, 21].

In Hong Kong, an optical fibre sensor system was deployed as a structural health monitoring system on a passenger rail system, with sensors attached to the rail and to the underside of carriages, measuring temperature and static and dynamic strain at critical locations. The system provided information on the loading of the passenger cars, deformation of the rails and of the carriages, temperatures of axles and brakes, and axle vibrations allowing assessment of corrosion and bearing wear $[22,23]$.

FBGs have been used to detect wheel flats [24] and have been mounted on a Maglev guide way to facilitate the determination of local changes in curvature and vertical deflection in response to the passing vehicle. The frequency components present within the FBG sensor measurements matched those of an accelerometer, and the deflection and curvatures determined from the measurements matched theoretical predictions $[25,26]$.

The measurement of the contact forces between the pantograph and catenary is an application in which the electromagnetic interference immunity of fibre optic sensors offers a significant advantage. The use of an OFS to measure pantograph contact loads has been evaluated in a laboratory environment, in which the loading system was capable of replicating the loads experienced at different train speeds, and under acceleration and deceleration. The system was shown to be capable of measuring quasi-static loads (up to $20 \mathrm{~Hz}$ ), and of measuring higher frequency components, which matched those detected by an accelerometer mounted on the pantograph. The measurement of dynamic loads in the vertical and horizontal directions between the pantograph and catenary on a high speed track and in mountainous regions has been achieved using FBG sensors embedded within the composite carbon/aluminum collector strip $[12,27,28]$.

Integrating FBG sensors into current collectors has been reported, in order to turn the pantograph into a 3 point bending sensor in direct contact with the overhead cable. This arrangement results in a reduction in sensitivity of the measurements to inertial forces introduced by the pantograph suspension structure. Using an interrogation system with a $500 \mathrm{~Hz}$ update rate, measurements undertaken on the TGV duplex between Paris and Vendome, with a speed of $320 \mathrm{~km} / \mathrm{h}$, allowing contact forces and current collector temperature to be computed in real time [29].

An array of FBG sensors installed on a short span railway bridge was found to be capable of the determination of train speed and weight distribution. The FBGs were mounted in a weldable package that was spot-welded to the track. Sensors were configured and measurements made such that it was possible to determine speed, acceleration and weight distribution of a passing train [Error! Bookmark not defined.]. The use of rail-mounted FBGs to detect and determine train types, axles, speed and acceleration, wheel imperfections and dynamic load calculations on a high speed line has been demonstrated [30Error! Bookmark not defined.]. Vibration signals induced in surrounding buildings by 
tram and train traffic has also been investigated using a commercially available FBG interrogation system with FBG based accelerometers [31].

\section{FBG instrumentation}

The FBG sensor interrogation system developed for this work is shown in figure 1. The output from a wavelength tuneable laser source (Santec HSL2000), was coupled into a network of directional couplers (fibre optic beamsplitters) to allow 4 arrays of sensors to be monitored simultaneously. The output wavelength from the laser was tuned rapidly through the optical spectrum (1262.5 nm -1311.5 nm) at a frequency of $2.5 \mathrm{kHz}$, capturing complete information on all of the FBGs in one sweep, resulting in a Nyquist limit of $1.25 \mathrm{kHz}$. The reflected signals were directed to photodetectors (Thorlabs PDA10CS). The output from the photodetectors were input to a high speed $(2 \mathrm{GS} / \mathrm{s}$ ) data acquisition system (National Instruments (NI PXI 5152 and NI PXI 1033), and the spectra were recorded, analysed and stored on a PC. The laser wavelength scan is linear to better than $1 \%$ over the scan range, with the start of the scan indicated by a TTL trigger signal. To reduce the effects of jitter of the trigger, a temperature stabilised FBG was included in one of the arrays and all of the measured Bragg wavelengths were referenced to this. As configured, the system offers a wavelength resolution of $1 \mathrm{pm}$ with a corresponding strain resolution of $\approx 1 \mu \varepsilon$. The FBGs were fabricated using the phase mask technique [32] in single mode optical fibre, that had been hydrogen loaded to increase its photosensitivity [33]. The FBGs were subsequently annealed at a temperature of $100{ }^{\circ} \mathrm{C}$ to ensure that the Bragg wavelengths were stable [34]. The FBGs were multiplexed in the wavelength domain. The quiescent Bragg wavelengths of the FBGs in each pair of arrays (1 and 2, and 3 and 4 in figure 2) were selected with consideration to the anticipated load at each measurement condition, to ensure that they would not overlap during operation and that thus there was no crosstalk. This was achieved by ensuring a minimum wavelength separation of $2 \mathrm{~nm}$ between adjacent FBGS in the wavelength domain.

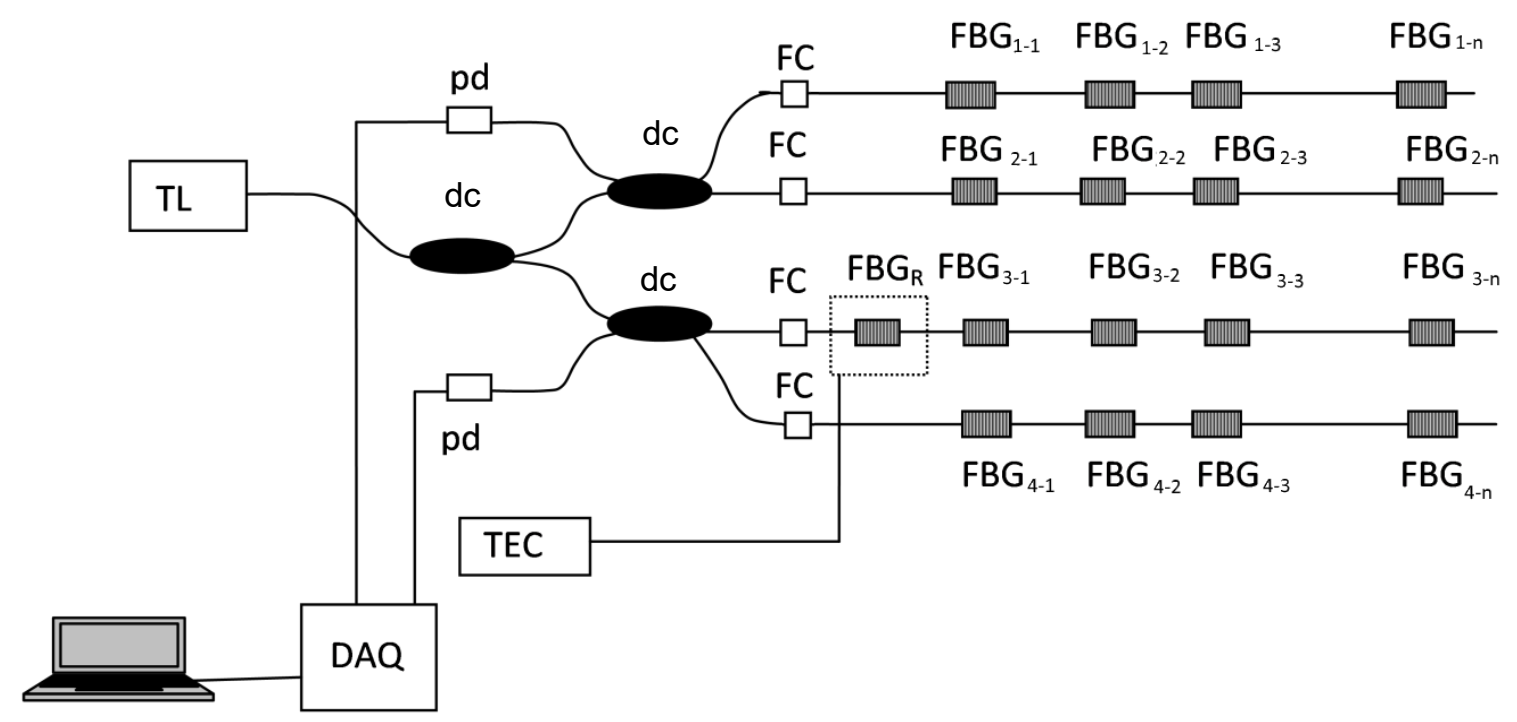

Figure 1. Schematic diagram of the FBG interrogation unit. TL, tunable laser; pd, photodiode; dc, directional coupler; FC, FC/PC fibre optic connector; FBG, fibre Bragg grating; TEC, thermoelectric cooler controller; DAQ, data acquisition card.

The system has been trialed on fishplated joints, switchblades and a stretcher bar on a busy city tram network in the UK. The Stagecoach Supertram network operates 25 three-car trams in the city of Sheffield. Each tram is equipped with four 2-axle bogies, each with their own drives.

\section{Sensor Installation}

The surface of the component was abraded at the positions at which the FBGs were to be attached to remove surface rust and to produce a clean, flat surface. The prepared surface was then cleaned using a 
solvent (isopropyl alcohol) and the sections of the optical fibre containing the FBGs aligned appropriately with respect to the longitudinal axis of the rail. The fibre was held in position temporarily using strips of adhesive tape at either end of each FBG. The FBGs were not pre-strained A thin layer of cyanoacrylate adhesive was applied and an accelerator was sprayed on to aid the curing of the adhesive. The FBGs were protected by covering with a two-part epoxy (Araldite). Once the epoxy had cured, the entire length of the fibre was coated with a silicone sealant to offer further protection. Comparison of the spectra recorded before and after deployment of the FBGs did not show any measurable bonding induced birefringence. The proximal end of the optical fibre was terminated with an FC/PC fibre optic connector, and the proceeding $1 \mathrm{~m}$ length was sheathed within a $3 \mathrm{~mm}$ outer diameter PVC protective jacket that contained an internal Kevlar thread. The process is illustrated in Figure 2 for sensors installed on a switchblade at a Supertram depot.

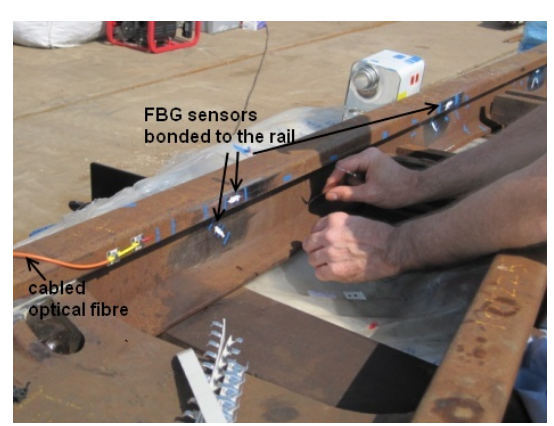

(a)

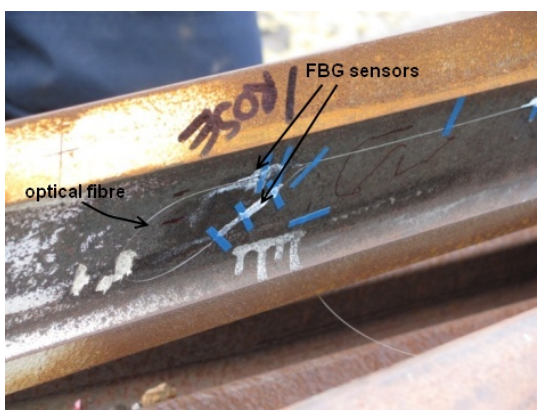

(b)

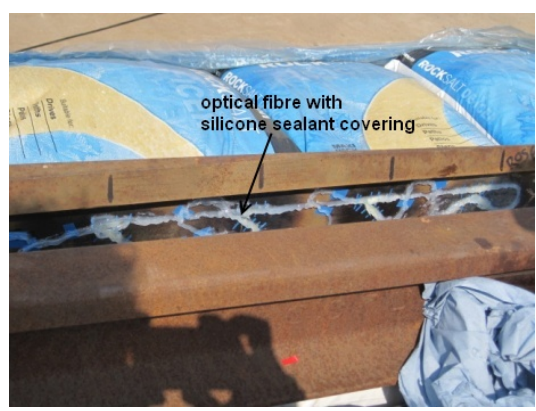

(c)

Figure 2. Stages in the attachment of the sensors to the switchblades (a) and (b) routing of the fibre and attachment of the sensors and (c) protection of the sensors using silicone sealant.

\section{Experiments and Results \\ 5.1. Fishplated Joints}

Fishplated joints are critical components on jointed rail track and the malfunction of such components can lead to catastrophic failure [15]. A fishplate is a metal bar that is bolted to the ends of two rails to join them together. The top and bottom edges are tapered inwards so the device wedges itself between the top and bottom of the rail when it is bolted into place. Failure of fishplate joints is generally a result of either cracking of the fishplate or loosening of the bolts, and such failures have been implicated in derailment of trains [15].

Two fishplate designs have been investigated during this project. For in-service monitoring a heavy-duty fishplate was instrumented, while for an initial laboratory based study a lightweight design was used. The fishplates and locations of the sensors are shown in Figure 3. FBGs sensors were bonded to the surface of each fishplate using a cyanoacrylate adhesive, and the sensors were then coated with a layer of epoxy resin to offer protection against mechanical damage. The sensor configuration on each of the fishplate surfaces is shown in figure 4(a). Two FBG arrays were bonded to each plate. One array with 5 sensors was mounted on each fishplate, with a sensor located below each bolt hole and with one at the mid-point of the plate. These sensors were oriented to measure the strain along the horizontal axis. The second array contained a sensor for temperature measurement and a further 2 sensors to complete a rosette arrangement below hole 3 . The sensors were located on the inner surface of the fishplates. The optical fibre downlead to each array was then sheathed in stainless steel armoured cabling to protect the optical fibre. 


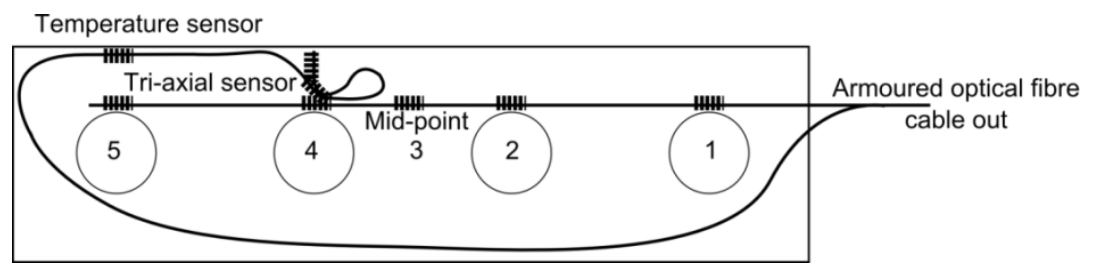

(a)

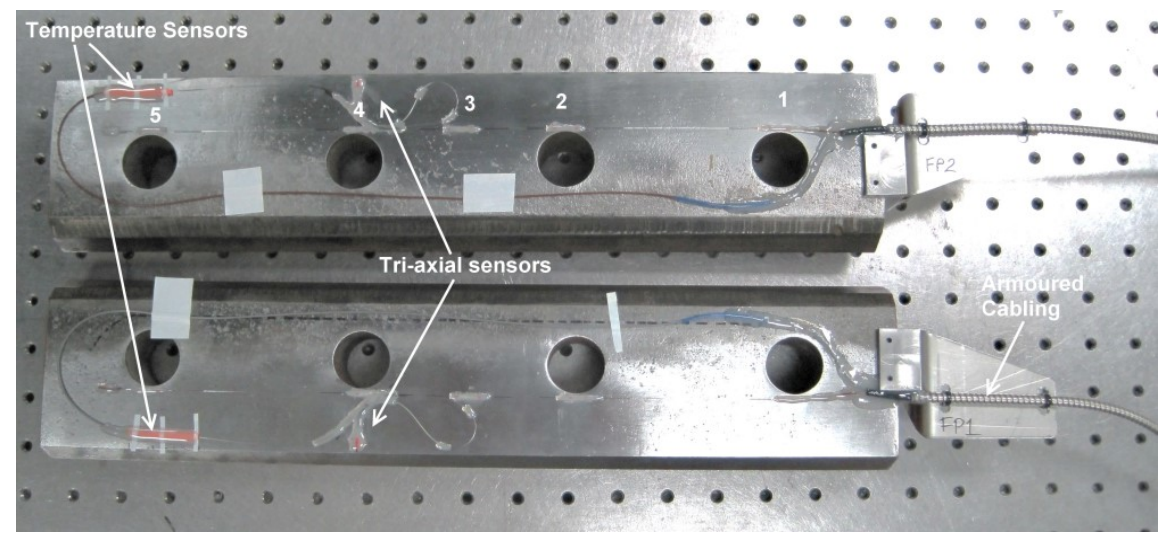

(b)

Figure 3. (a) Schematic illustrating the location of the optical fibre Bragg grating strain and temperature sensors [14]. (b) Photograph of the instrumented fishplates [14. The dimensions of the fishplates are $40 \mathrm{~mm} x$ $90 \mathrm{~mm} \times 400 \mathrm{~mm}$.

The second fishplate, used in the tests undertaken in laboratories at Tata Steel's Swinden Technology Centre, is shown in figure 4. The location of the sensors, shown in figure 4(b), was informed by the output from an FEM model, shown in figure 4(c) and 4(d). The model indicated a high strain gradient near the bolt holes. Using the ability to form FBGs with short gauge lengths and the ability to fabricate an array of FBGs in series in the fibre with small physical spacing, the array indicated in figure 4 (b) was fabricated. The FBGs were each of length $2 \mathrm{~mm}$, and under the 2 central holes the adjacent sensors were spaced by $2 \mathrm{~mm}$.

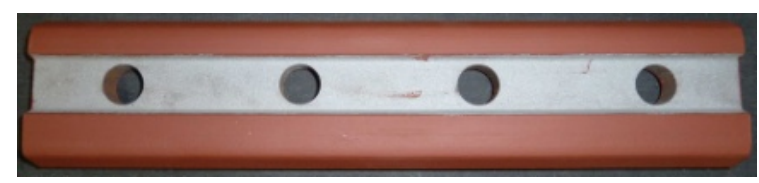

(a)

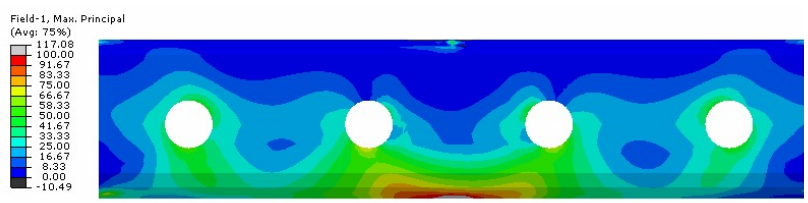

(c)

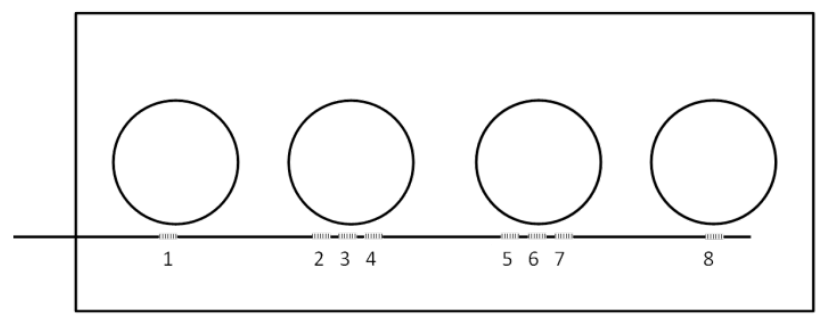

(b)

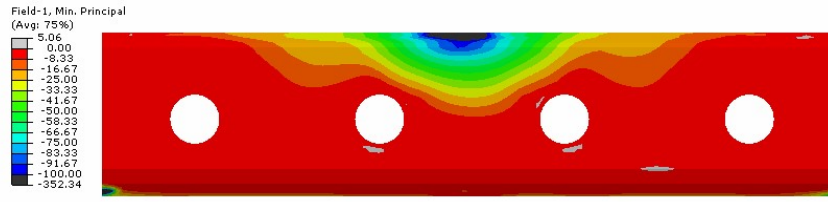

(d)

Figure 4. (a) The fishplate (b) the location of the FBG sensors, where the numbers are used to identify the individual sensors. (c) Maximum principal strain and (d) minimum principal strain distributions predicted by finite element modelling. 


\subsubsection{Laboratory testing}

FBG sensors were used to monitor the response of a fishplate joint to loading on mechanical testing systems in a laboratory environment. The aim of this trial was to validate FE models of the joints, and to assess if such testing could be used to inform the analysis of the data obtained from on-track trials of the fishplates. The fishplate, shown in figure $4(\mathrm{a})$, was attached to two $2 \mathrm{~m}$ long rail sections, and the system was mounted in a 4 point bending configuration, as shown in figure 5 . The fishplate was subjected to step increases in load and to sinusoidal, cyclic loading.

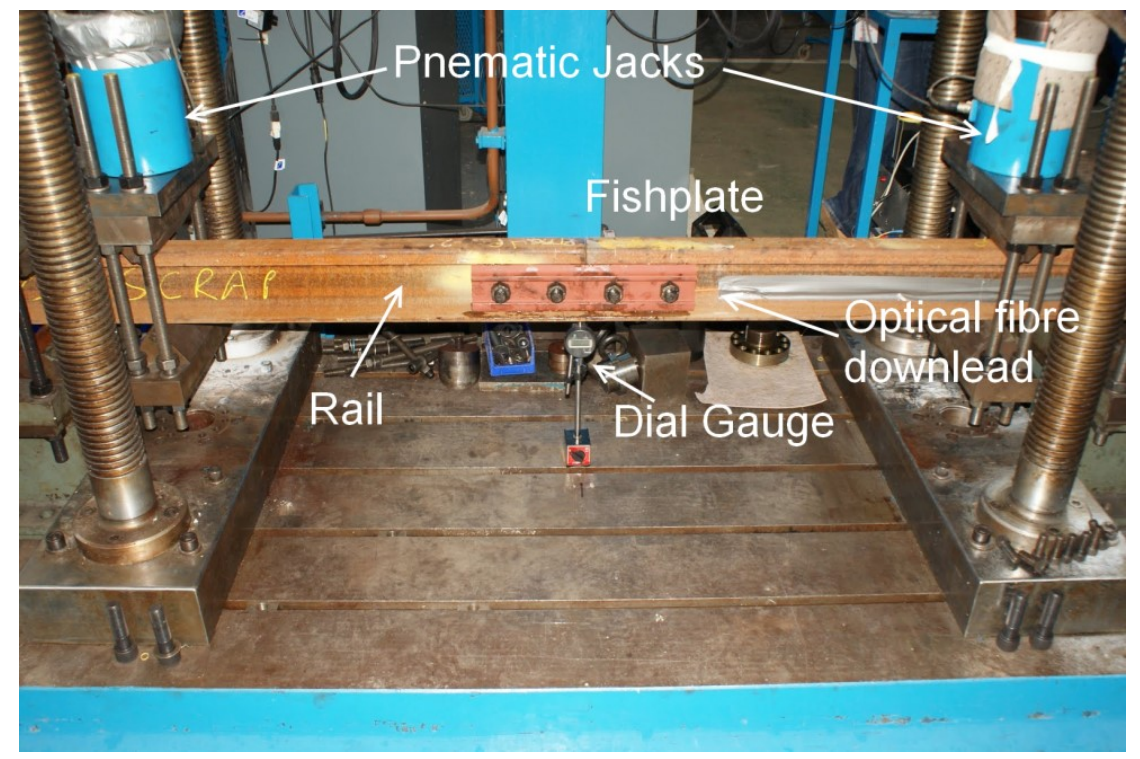

Figure 5. The 4-point bend configuration used to test the FBG- instrumented fishplated joint.

Figure 6 shows the peak strain measured by the FBG arrays for a load applied equally to the hydraulic rams in the 4 point bending configuration using a sinusoidal waveform at $5 \mathrm{~Hz}$ with $40 \mathrm{kN}$ peak to peak amplitude (simulating loading that would be induced by the passage of the tram). Similar results were obtained when the load was applied in a step-wise fashion. Figure 6 (a) shows that the FBGs are able to resolve the strain gradient that is present in the FEA analysis show in figure 4(c), indicating qualitative agreement with the model. Differences between the experimental configuration and the previously undertaken FEA preclude direct correlation of simulation and experiment. Figure 6 (b) shows that, as the bolt-torque on the left-hand inner bolt (adjacent to sensors 2, 3 and 4) increased, the measured strain decreased, with the sensors adjacent to the under-torqued bolt exhibiting the largest reduction in strain. 


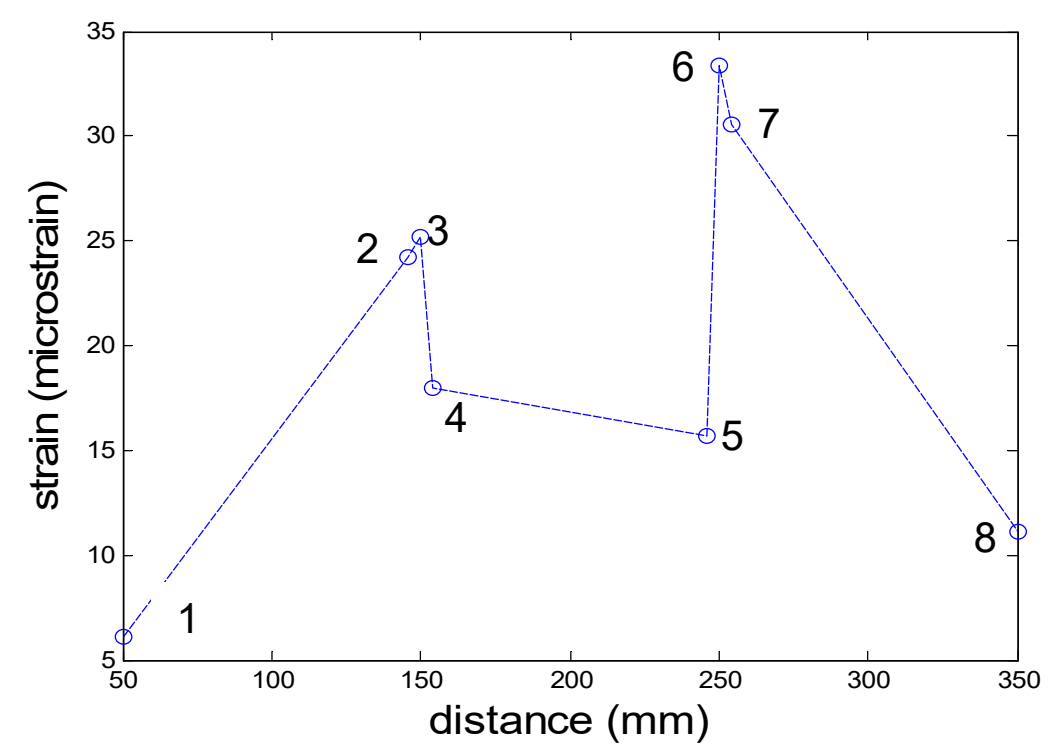

(a)

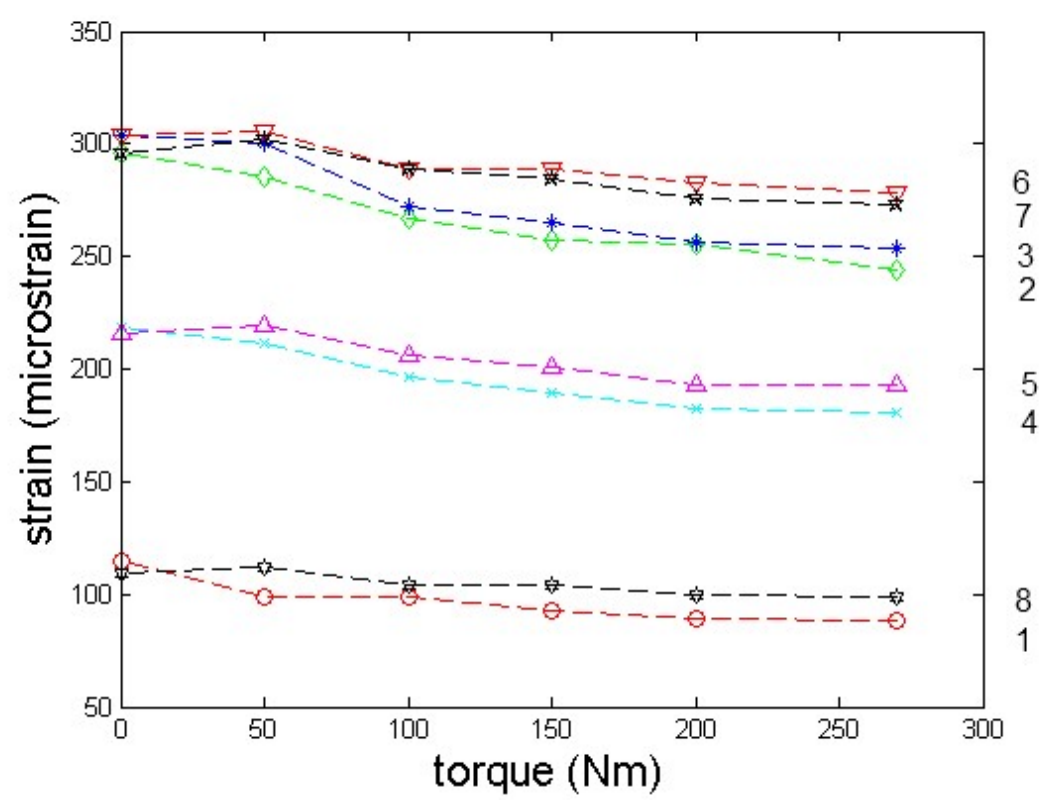

(b)

Figure 6. Results from the 4-point bending of a fishplate joint. (a) The strain profile measured using a fibre optic sensor array (the dashed line is a guide to the eye, and the numbers indicate the sensors) when all bolts were at the same torque, $270 \mathrm{Nm}$. The locations of the sensors are measured relative to the left hand edge of the fishplate. (b) The strains measured for a $40 \mathrm{kN}$ applied load when the torque of the left-hand inner bolt (adjacent to sensors 2, 3 and 4) was varied. The numbers refer to the sensor locations, identified in figure 6 (b).

The joint was also excited using an instrumented impact hammer. The dynamic response exhibited a damped sinusoidal response at the clamped rail system resonance frequency of $80 \mathrm{~Hz}$. Although this is an artificial response, not related to in-track resonant behaviour, it is sufficient to show that at the lower end of the vibration range, the strain profile derived from the response matched that measured for the quasiDC load variation of the load, as shown in figure 7. 


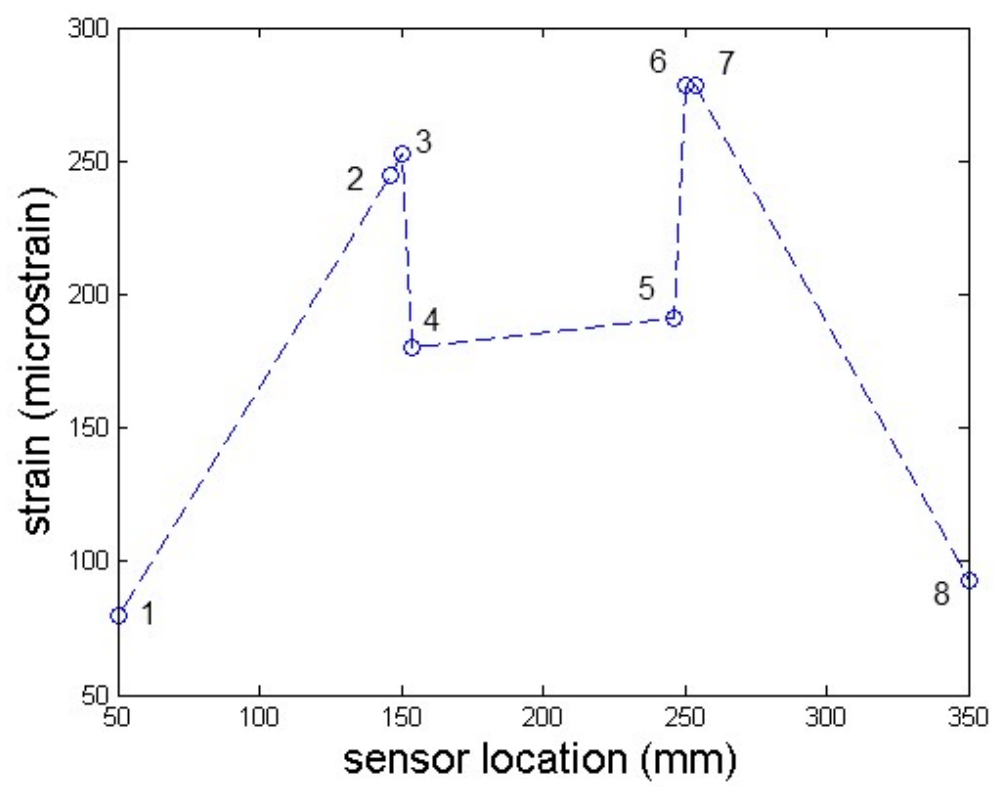

Figure 7. Strain distribution derived from the FBG sensor responses to dynamic excitation (impact hammer) of the fishplated joint with the bolts at a torque of $40 \mathrm{kN}$. The locations of the sensors are measured relative to the left hand edge of the fishplate.

The results presented in this subsection indicate that the measurement of the strain amplitude at different locations along the fishplate can provide information on the torque of individual bolts, and that the strain distribution obtained from static loading is the same as that under dynamic loading at the rail resonance frequency.

\subsubsection{On-Track trials}

A trial was undertaken to facilitate the acquisition of data under controlled conditions, whereby an unloaded tram would pass over an instrumented fishplated joint at constant speed. The instrumented fishplate shown in figure 8 was installed on the Supertram network at a location called the 'Meadowhall curve', a single track section with bi-directional traffic from and to the city of Sheffield, with a final tram stop at Meadowhall. Trams that were operating towards Meadowhall were termed outbound trams, and trams in the opposite direction, towards the city, were termed inbound. The instrumented fishplate is shown in situ in figure 8(c). The data acquisition system was placed $20 \mathrm{~m}$ from the plate location, connected to the instrumented fishplates via optical fibre cables, generally referred to as patch cords.

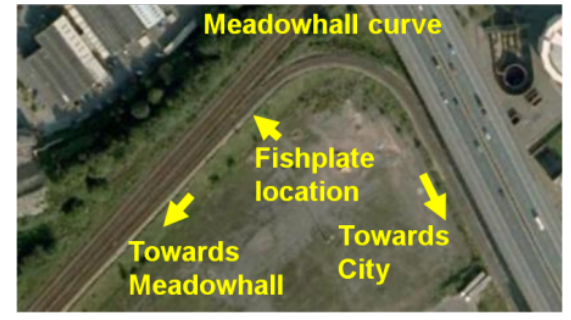

(a)

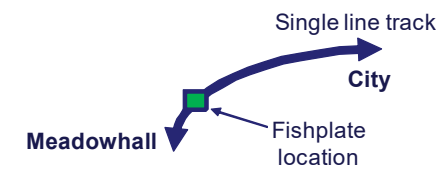

(b)

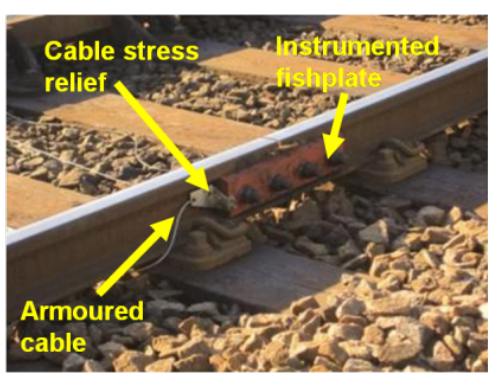

(c)

Figure 8. Aerial view of the field trial location, (a) simple schematic of track, (b) and photograph of instrumented fishplate joint in track (c) 14.

Data were recorded that captured the response of the fishplate to tram crossings at 4 different torque settings. Each crossing lasted approximately 7 seconds. One of the aims of this trial was to gain an insight 
into the mechanical behaviour of the fishplate under differing conditions and assess how that behaviour is detected by the fibre sensor. The different torque settings are detailed in table 1.

Table 1: Torque settings for fishplate trial

\begin{tabular}{|c|c|}
\hline Test & Torque \\
\hline 1 & $320 \mathrm{Nm}$ \\
\hline 2 & $270 \mathrm{Nm}$ \\
\hline 3 & $150 \mathrm{Nm}$ \\
\hline 4 & $0 \mathrm{Nm}$ \\
\hline
\end{tabular}

Figure 9 shows the strain measured by sensor 3 within the array in response to tram crossings in the Meadowhall bound direction, for bolt-torques of $320 \mathrm{Nm}, 270 \mathrm{Nm}, 150 \mathrm{~m}$ and $0 \mathrm{Nm}$. As the torque decreases, the high frequency spike apparent in the $1^{\text {st }}$ wheel crossing of each bogey decreases in amplitude and the ratio of the peak strain for the $1^{\text {st }}$ and $2^{\text {nd }}$ wheels on the bogey changes. There is a significant change in the form of the response with the bolt-torque set to $0 \mathrm{Nm}$.

$320 \mathrm{NM}$

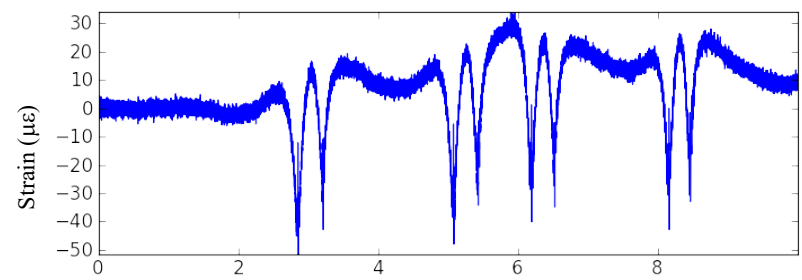

$270 \mathrm{Nm}$
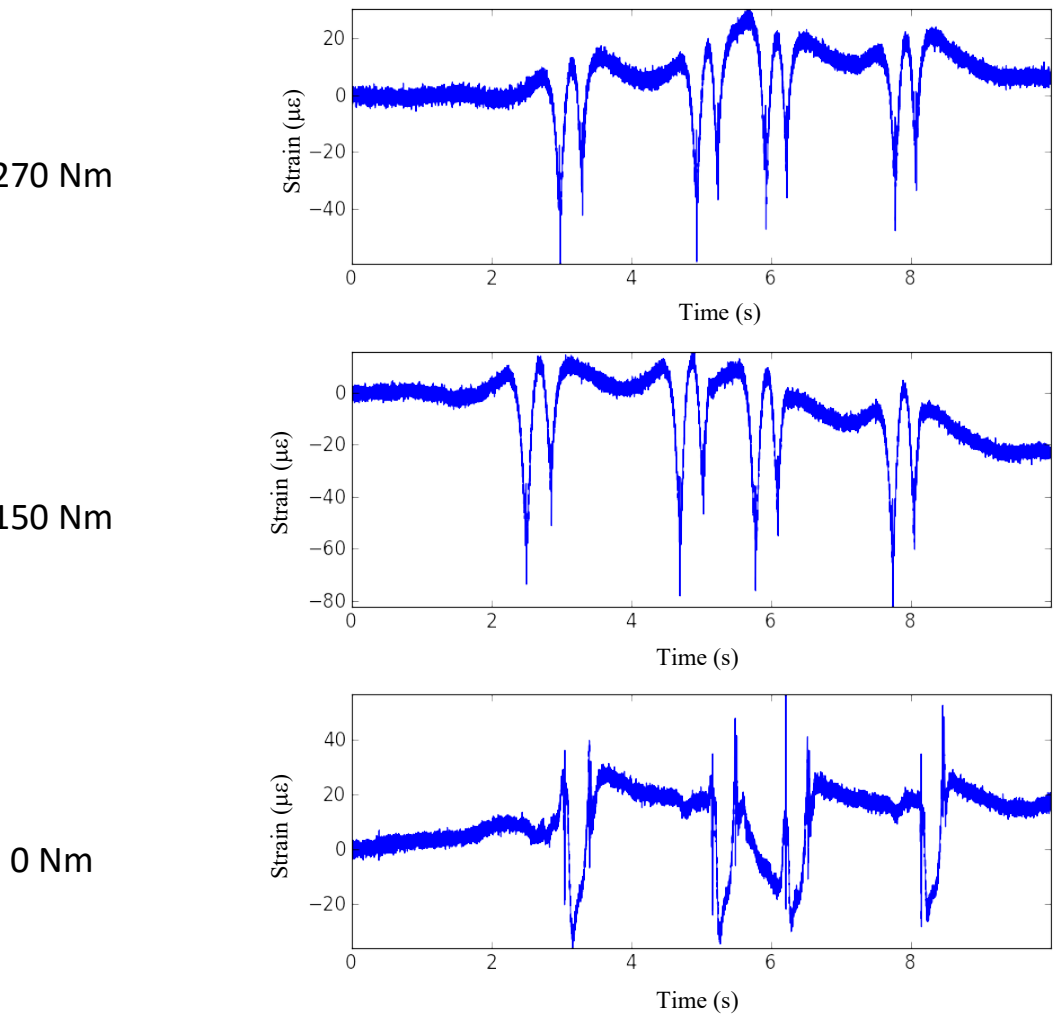

Figure 9. Strain signature measured using FBG sensor 3 (identified in figure 4) for trams crossing a fishplated joint at the Meadowhall curve on the Sheffield Stagecoach Supertram network, for fishplate bolt-torques that varied from $320 \mathrm{Nm}$ to $0 \mathrm{Nm}$. The results for $270 \mathrm{Nm}$ and $0 \mathrm{Nm}$ are reproduced from [14]. 
To investigate the dynamics underlying the time-series, wavelet transforms were used. Wavelets were used here because the technique enables the frequency content of transient data to be more accurately illustrated than is possible using conventional FFT methods. Wavelet analysis of time series allows the frequency content to be localized in time, a feature that is significant when dealing with non-stationary signals, allowing the time-dependent variation of the characteristic frequencies to be extracted. When wavelet analysis is combined with the statistical processing method of principal component analysis, together with automated pattern recognition, it becomes possible to map the changing and complex pulse response to wheel passes for different structural states.

Each time series was subjected to a multilevel discrete wavelet transform (DWT), using the PyWavelets module for the Python programming language [35], to allow the calculation of the energy contained in a number of different frequency bands (or DWT levels). The reverse biorthogonal 3.1 wavelet was selected [40]. Principlal component analysis (PCA) [36] and a support vector machine learning algorithm [37] were used to analyse the relationship between bolt condition and strain signature and to classify subsequent data using the mlpy module for the Python programming language [38], allowing the data to be mapped onto a space in which the component of the data with the largest variance is plotted against the component with the $2^{\text {nd }}$ highest variance, and which is orthogonal to the $1^{\text {st }}$ component. Here, as is common, the choice of wavelet was based on a qualitative, visual matching of the shape of the wavelet to the signal, although there is considerable interest in providing a qualitative means for wavelet selection [39].

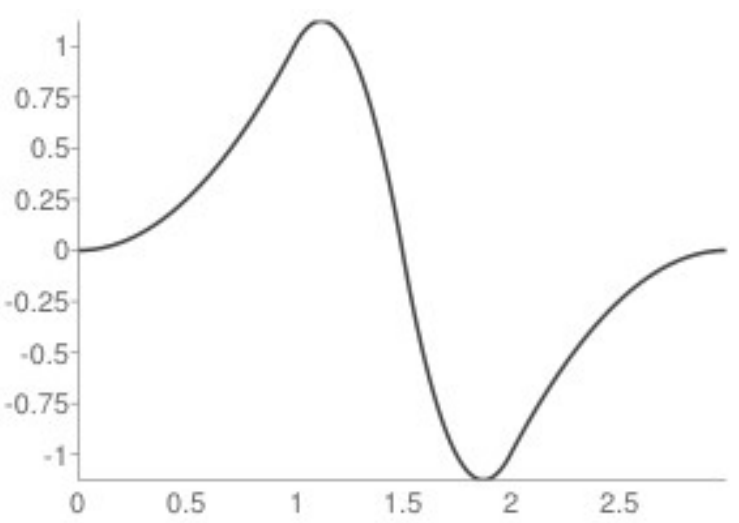

(a)

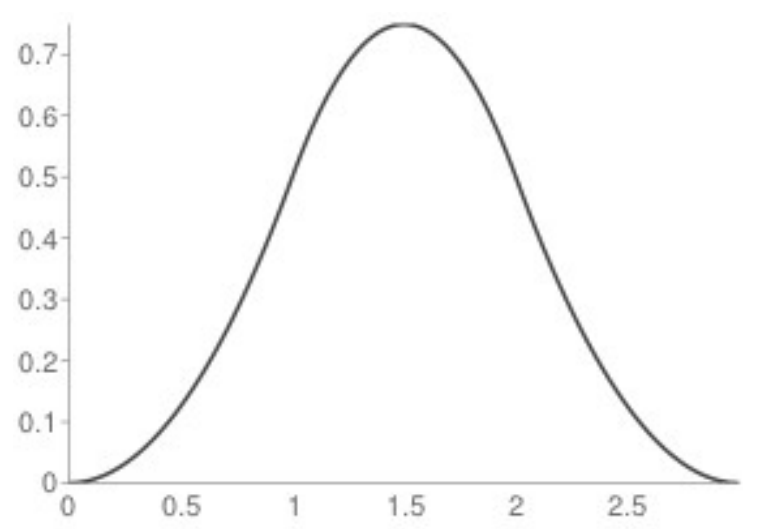

(b)

Figure 10. Reverse bi-orthogonal 3.1 wavelet (a) and its scaling function (b)[40]

A support vector machine (SVM) algorithm form the mlpy Python module [Error! Bookmark not defined.] was used to learn and categorize patterns in the data. The classification and clustering of the data are illustrated in figure 11 and 12 . The results in figures 11 and 12 represent the changing ratios of energy levels in different frequency bands, as bolt torques in the bolts are changed - the important point being that the different states are very well differentiated as different locations in the diagrams. The results suggest that it is possible to classify the strain signatures corresponding to trams crossing a fishplate with varying bolt-torque by considering the frequency content of the measured strain. 


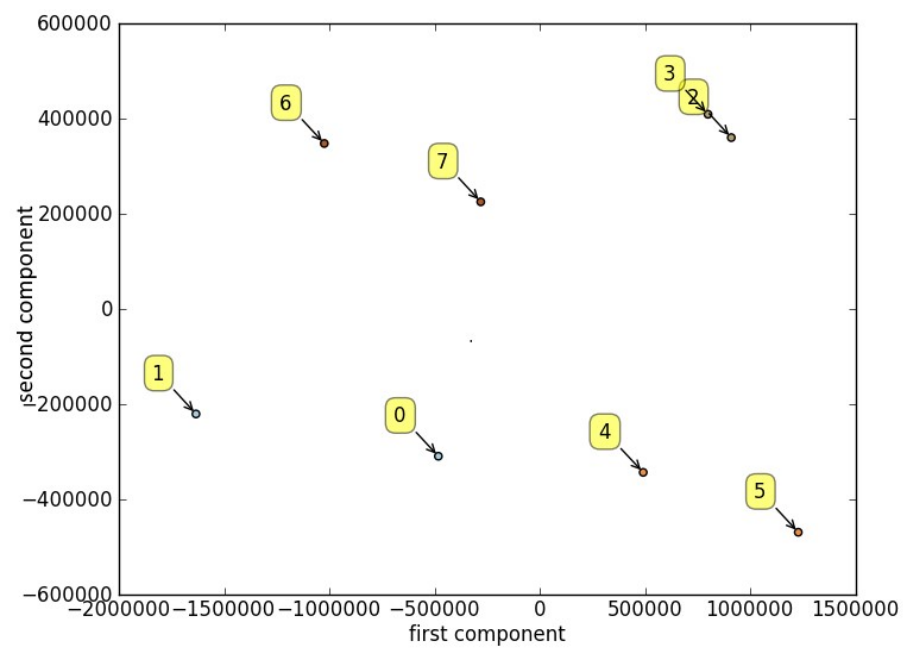

Figure 11. PCA plot derived from the training data for a sensor mounted between the central bolt holes of a fishplate deployed on the Meadowhall curve of the Sheffield Stagecoach Supertram network. The numbers indicate the file number analysed with files 0 and 1 obtained from separate crossings of a tram in the same direction for bolt-torque of $0 \mathrm{Nm}$, files 2 and 3 obtained with a bolt-torque of $150 \mathrm{Nm}$, files 4 and 5 with a bolt-torque of $270 \mathrm{Nm}$ and files 6 and 7 with a bolt-torque of $0 \mathrm{Nm}$.

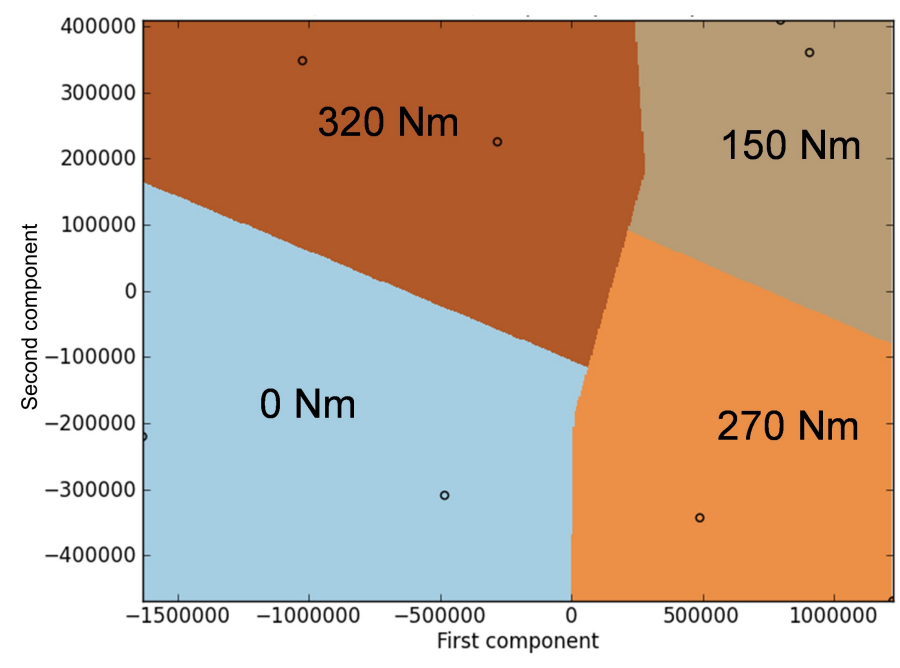

Figure 12. The output from the support vector machine (SVM) analysis of the PCA data, where the coloured regions indicate the extent of the PCA space into which signals derived for a tram crossing with bolt-torque indicated would map.

\subsection{Switchblades and Stretcher bars}

FBG sensor arrays were mounted on 4 switchblades while they were stored in the yard at the Supertram Depot. The two switches were subsequently installed, in April 2011, at a location known as the "Delta". The installed switches are shown in figure 13. Each switchblade had the same sensor configuration, as shown in figure 14. For convenience, each blade had two sensor arrays, referred to as the body and nose arrays, as indicated. Following the installation of the switches at the delta, FBGs were also attached to a stretcher bar with the stretcher bar in-situ, as shown in figure 15. The sensor arrays were monitored periodically, recording the response of the components to the passage of a number of trams of an 8 hour period, approximately once every 6 weeks. 


\subsubsection{Switchblades}

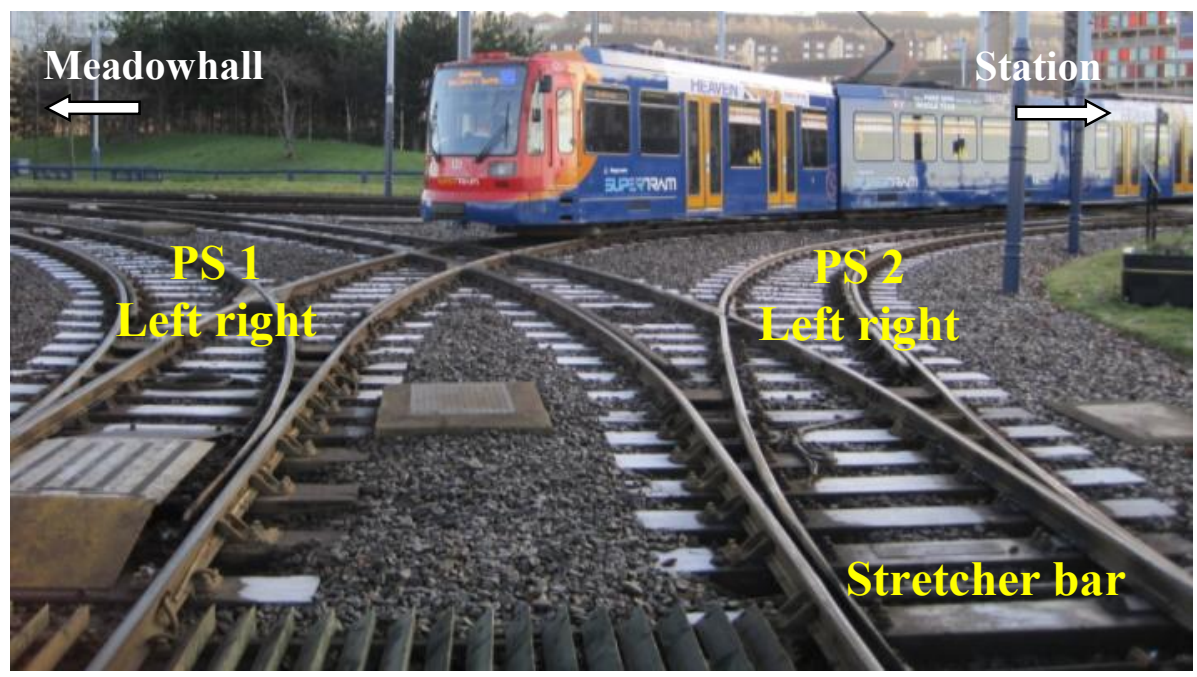

Figure 13. Configuration of the instrumented switchblades and stretcher bar on the Delta. The photograph was taken looking from the city bridge. "Meadowhall" and "Station" are significant stations on the network, and are included to allow the direction of travel of the crossing tram to be distinguished. "PS1 Left", "PS1 Right", "PS2 Left" and "PS2 Right" are used to identify the switch blades in the text. .

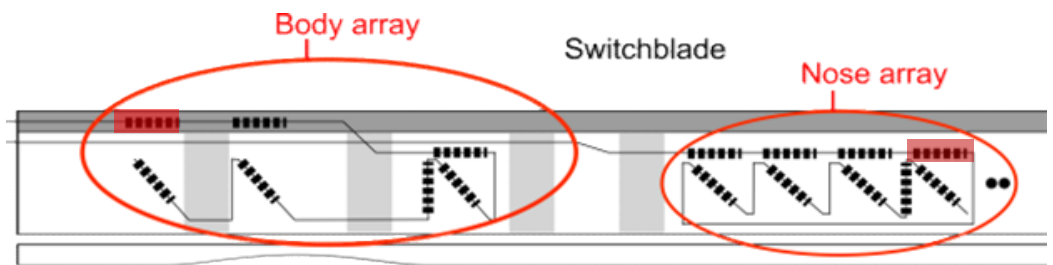

Figure 14. FBG sensor array configurations attached to each switchblade. The responses of the highlighted sensors are discussed in the text [14].
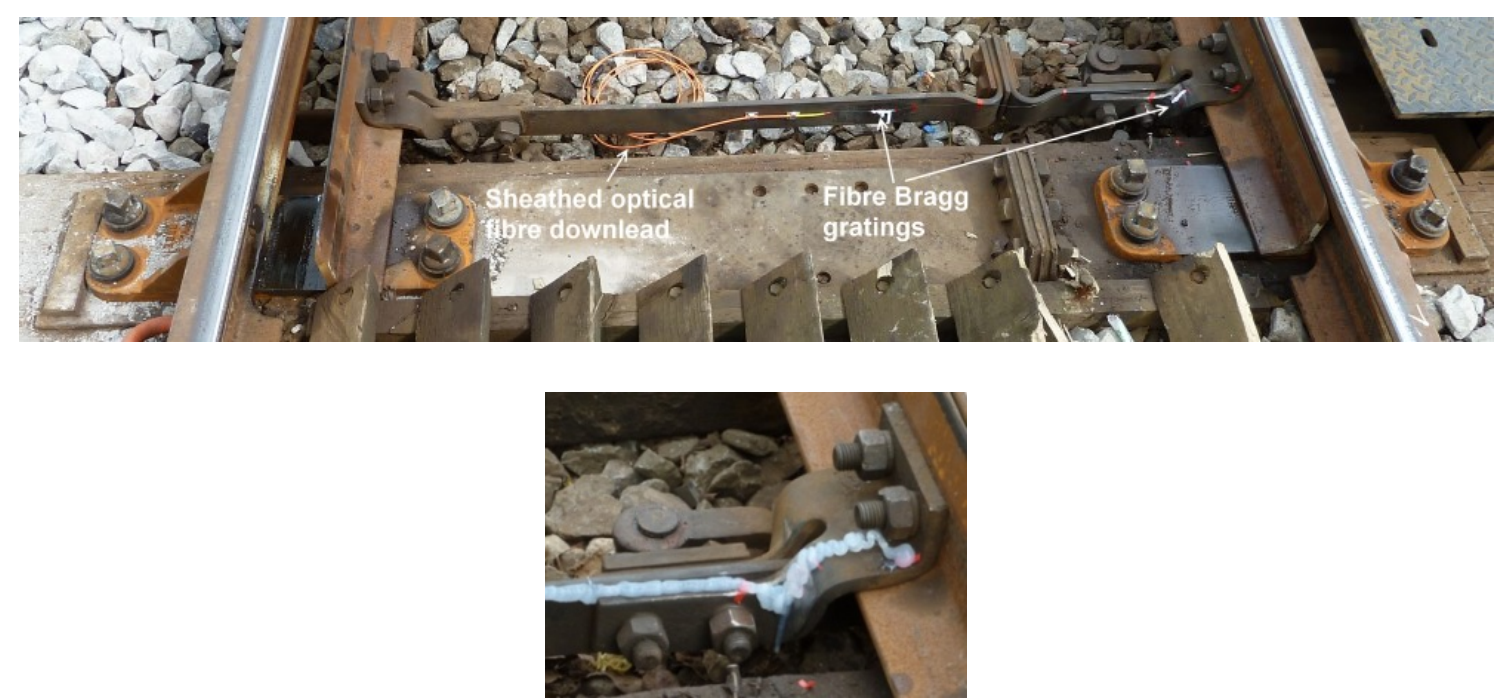

Figure 15. Images showing the placement of sensors on the stretcher bar and subsequent sealant protection. 
As an illustration, the evolution of the strain signature from one of the sensors, attached to the left-hand switch blade of PS1 (PS1 Left, identified in figure (14), is shown in figure 17. The location of the sensor is indicated in figure 15 is highlighted in the "nose array".

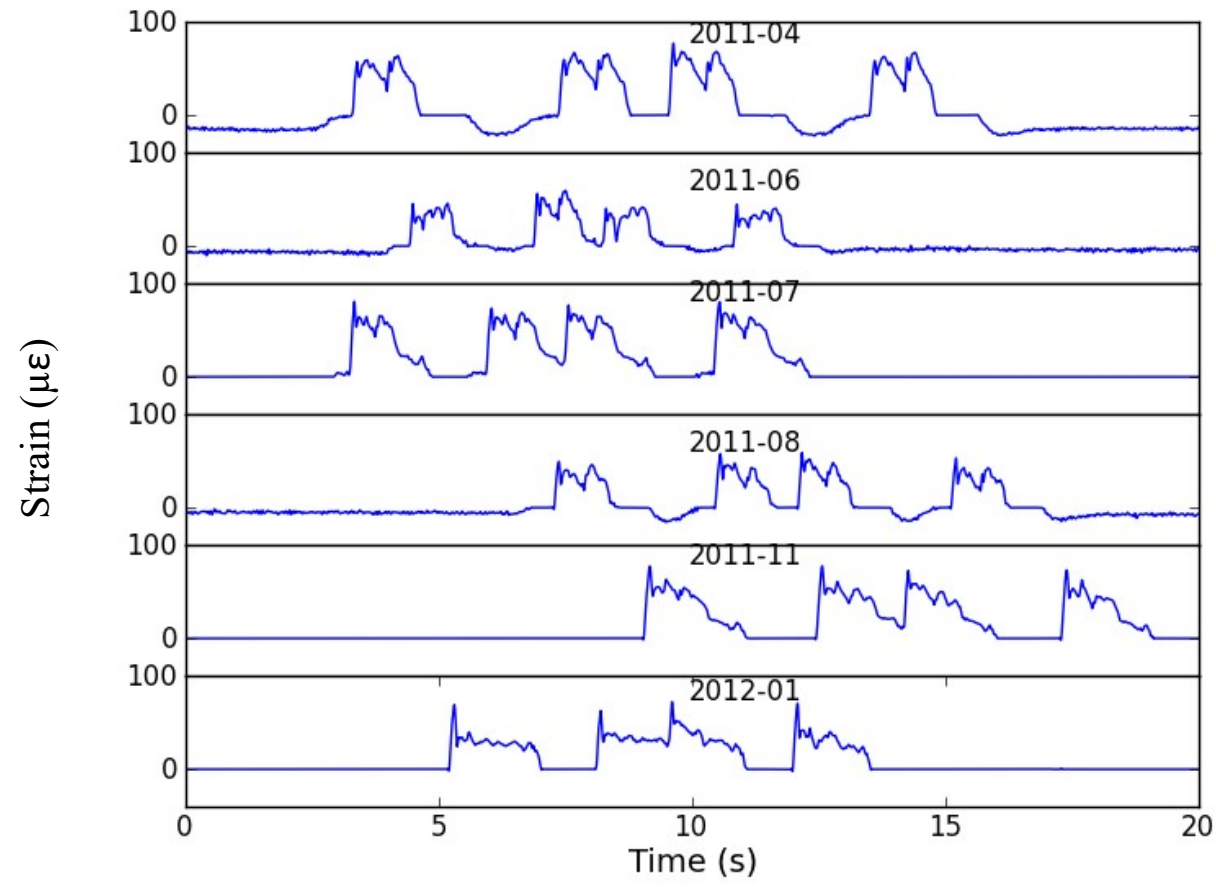

Figure 16. Evolution of the strain measured by a sensor on PS1 Left, identified in figure (14), during the passage of a tram. The month in which each trace was recorded is indicated in the figure. The trams were crossing the switch from the station direction.

A qualitative analysis of the strain signatures recorded for trams passing over the switch during a given field trial indicated that the signatures were repeatable, and that the loading of the tram had no significant influence on the form of the signature. Figure 16 shows that the form of the signal did vary over the 9 months following the installation of the switch. Immediately after installation of the switchblade in April 2011 it was possible to distinguish individual wheel crossings and to separate the strain induced by bogies 2 and 3. During the following months the spike at the start of each wheel crossing increases in amplitude, and it is apparent in November 2011 and January 2012 that the crossing of the wheels on a single bogey become less distinct and the reaction to the crossing of bogey 3 starts before the rail has recovered from the passing of bogey 2 . 
$01 / 2012$

$11 / 2011$

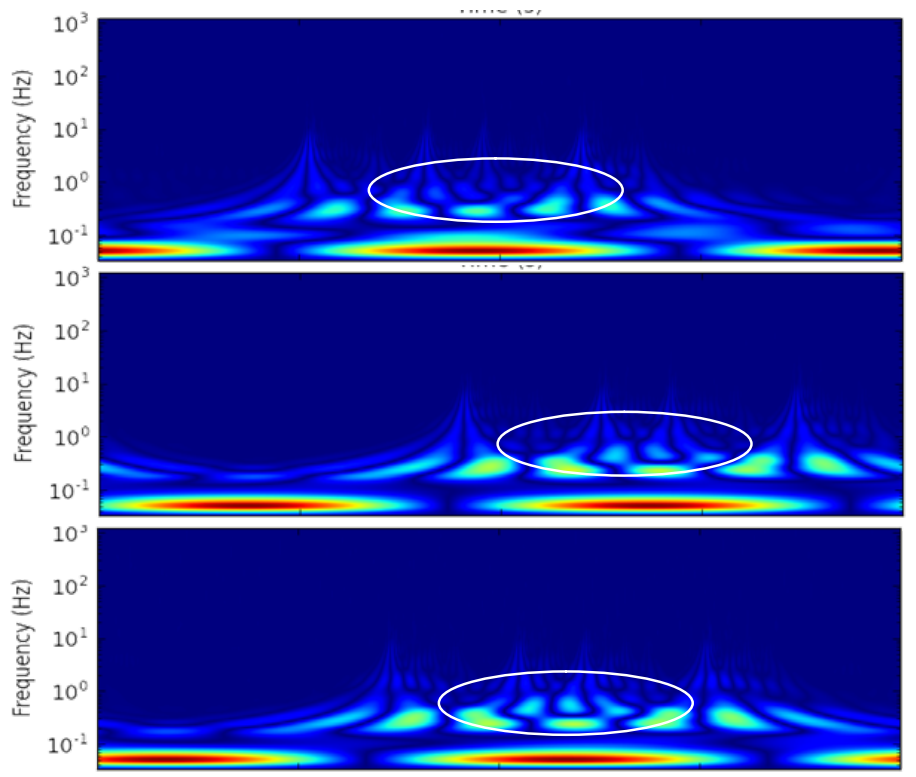

$07 / 2011$

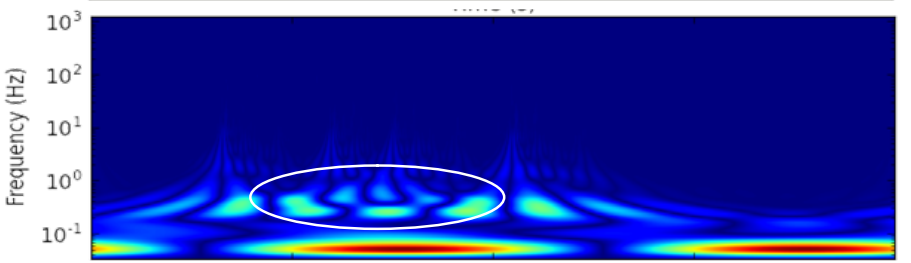

$06 / 2011$

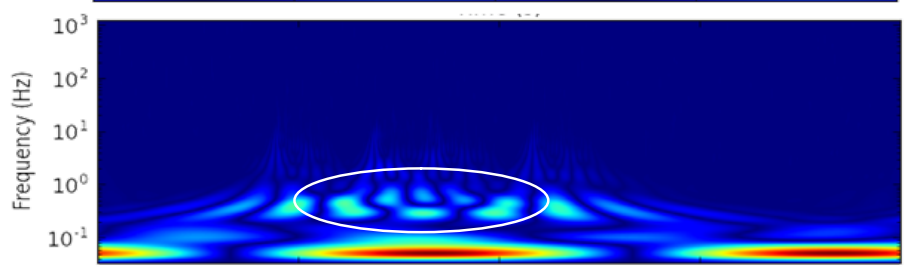

$04 / 2011$

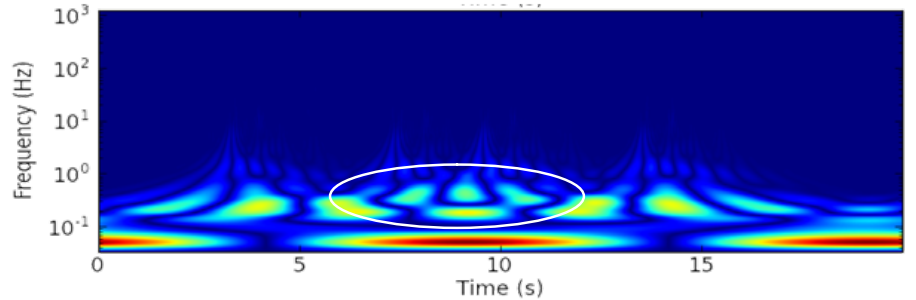

Figure 17. Continuous wavelet transform (CWT) of the data presented in figure 17, using a Morlet wavelet. The white ellipse highlights the region of interest within the CWT. The colour scale is such that red corresponds to the maximum value of the CWT and blue to zero.

The frequency content of this data was analysed using a continuous wavelet transform (CWT). A CWT is in essence equivalent to a multilevel DWT, where the discretization of the scale levels is on a finer, quasicontinuous, scale than the dyadic scaling described previously. The CWT allows the production of a timefrequency map, allowing the frequency content of the time series to be localized in time. The CWT of this data, calculated using a Morlet wavelet, is show in figure 17. The CWT reveals that features that lie in the equivalent frequency range of $100 \mathrm{~s} \mathrm{mHz}$ (highlighted by the white ellipse) show changes in energy content. The features highlighted in figure 17, which correspond to the envelope of the response to the crossing of bogeys 2 and 3, change over time, as expected from the qualitative analysis of the data presented in figure 16. The changes in this frequency range are shown more clearly in figure 18 . Similar trends are observed for the other sensors in the array. 

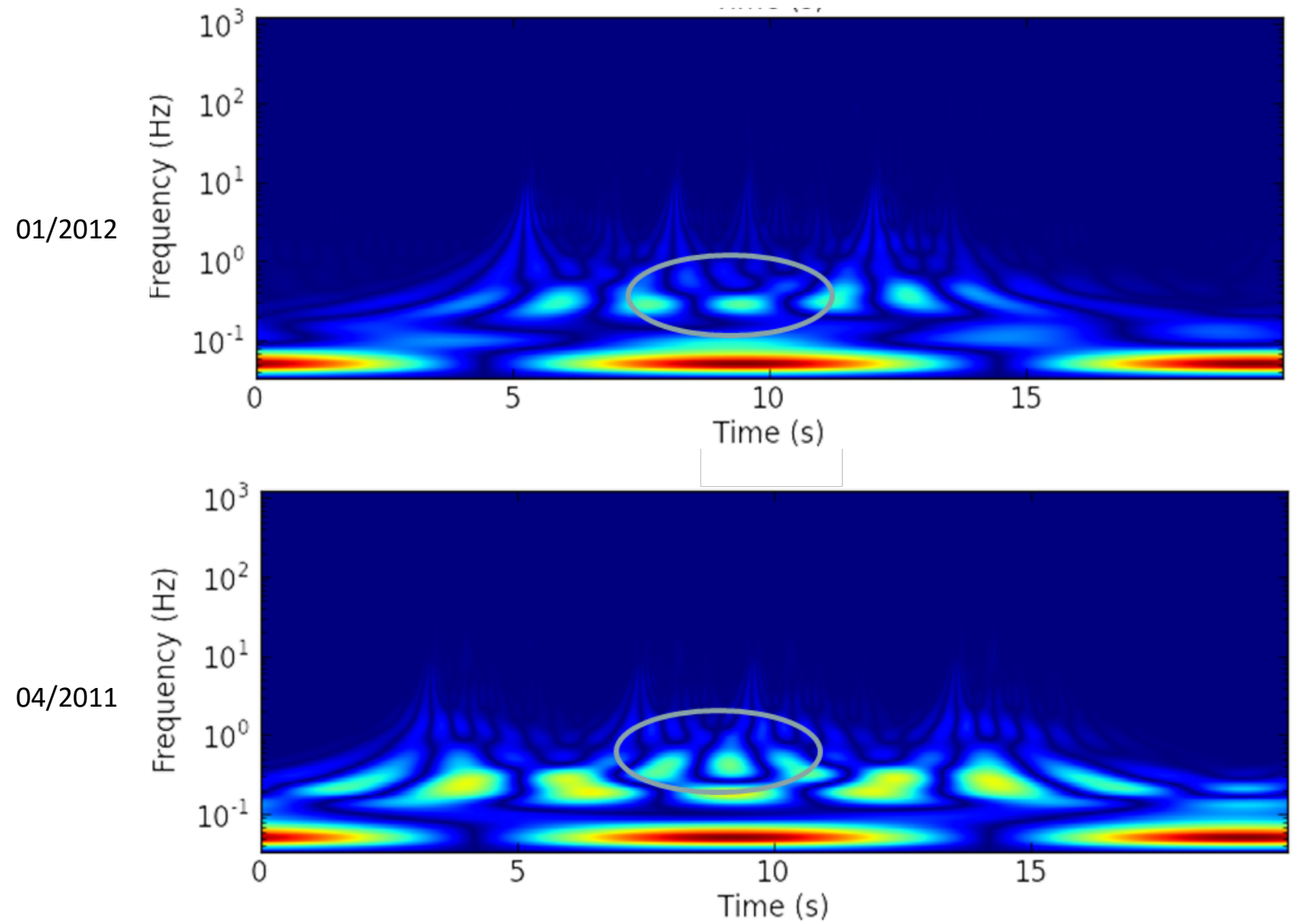

Figure 18. CWT of the strain measured by the sensor, highlighted in the "nose array" in figure 15, on PS1 Left in April 2011 and January 2012.

Further analysis of this data is on-going, with the aim of providing a quantitative measure of the change to the strain signature over time. A complicating factor is the variation of the speed of the trams crossing the switch, which influences the frequency of the CWT features of interest. As the speed of the tram can be calculated from the data, it is possible to plot the strain as a function of the distance travelled by the tram, which, provided the frequencies of the components of interest scale linearly with speed, should allow quantitative comparison of the CWT plots. An investigation of this approach is underway. Future work will explore the relationships of the data provided by the different sensors in the array with the aim of identifying further indicators of rail degradation, and on factors on interest, such as changes in the location at which the tram wheels contact the switch.

Other aspects of the switch were also monitored. When PS2 was installed on the network, the geometry was incorrect, resulting in large loads on the switchblades and stretcher bar, and noisy operation. In July 2011 the switch geometry was corrected using a crow clamp, as shown in figure 19 (a). The response of an FBG sensor attached to PS2 left, highlighted in figure 14 as the first in the 'body array', is shown in figure 19 (b) showing a large compressive load. The geometry correction made the operation of the switch much quieter. 


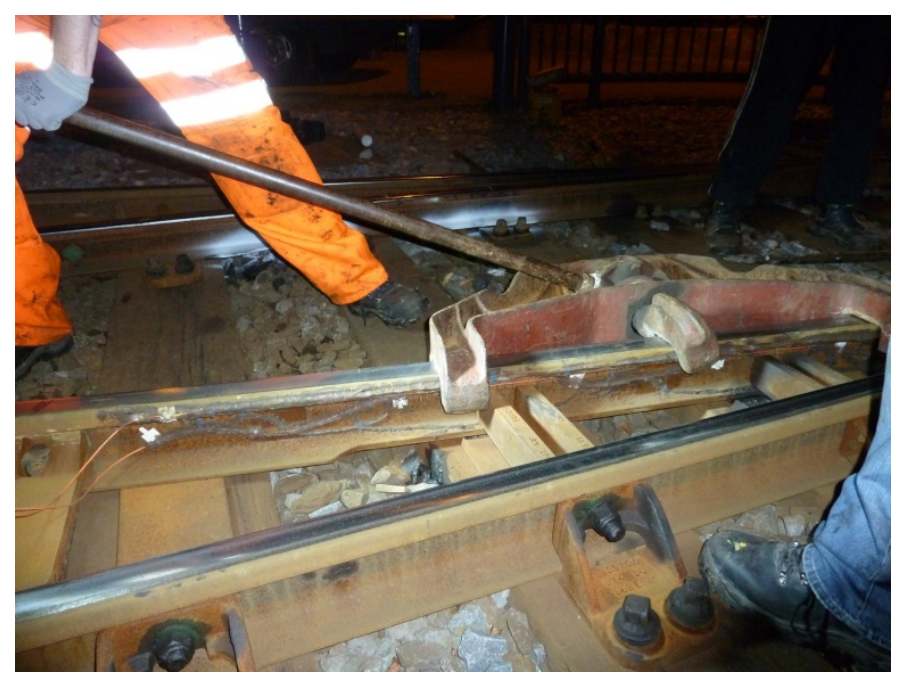

(a)

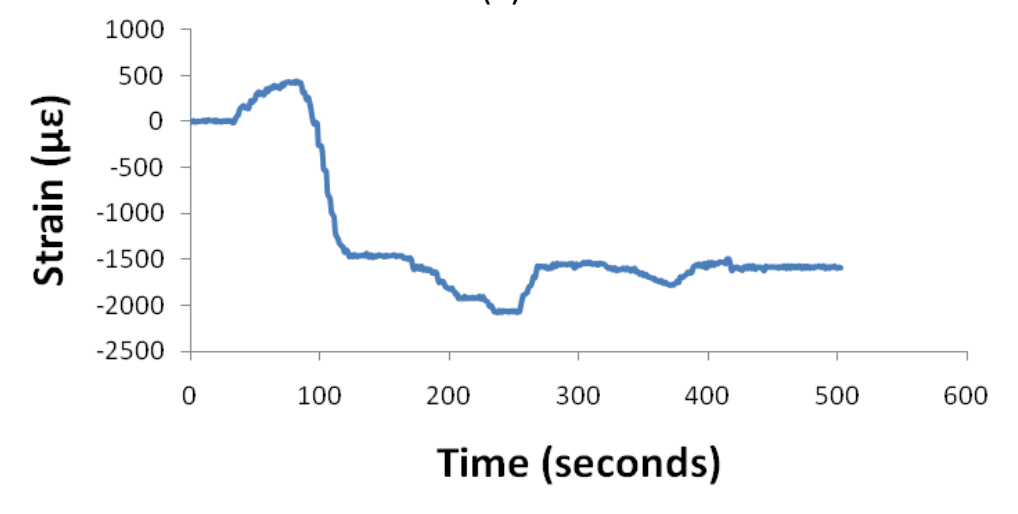

(b)

Figure 19. (a) Correction of switch geometry using a crow clamp, (b) strain measured by the $1^{\text {st }}$ FBG sensor of the 'body array' during geometry correction.

Whilst this is not the original purpose of the condition monitoring project using FBGs, it does show the ability to measure very high strains without loss of function, and suggests a possible contribution to a calibrated approach to in-track curved rail adjustments. The strain induced during operation of the switch PS1 was also monitored, an example is shown in figure 20, (recorded after the correction of the switch geometry).

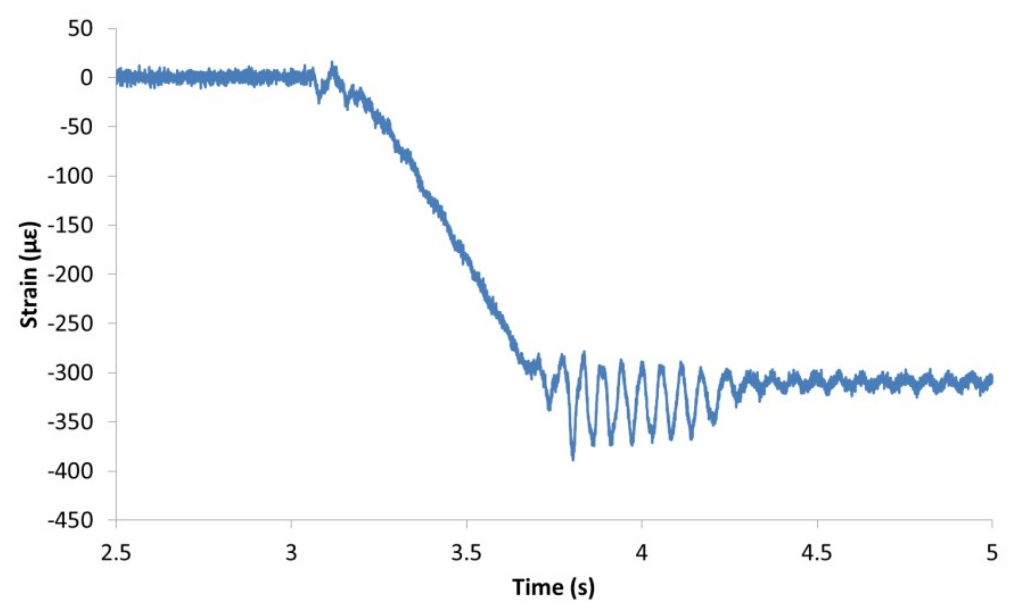

Figure 20. Strain measured during operation of the switch PS1. 


\subsubsection{Stretcher Bar}

Two sensors were mounted on the stretcher bar, as show in figure 16. The stretcher bar experienced significant changes in condition over the period of the trials, as summarised in Table 2.

\begin{tabular}{|l|l|l|}
\hline \multicolumn{3}{|l|}{ Table 2. Stretcher Bar Condition during the trials } \\
\hline Trial & Date & Notes on Stretcher Bar Condition \\
\hline 2 & $04 / 2011$ & newly installed, spring return connected \\
\hline 4 & $06 / 2011$ & cracked knuckle, spring return disconnected \\
\hline 7 & $08 / 2011$ & new knuckle and FBG sensor, spring return disconnected \\
\hline 8 & $11 / 2011$ & No maintenance-induced change \\
\hline 9 & $01 / 2012$ & No maintenance-induced change \\
\hline
\end{tabular}

Changes in the strain response of the sensor mounted on the knuckle, to trams bound for the Station and Meadowhall, are shown in figure 21 (a) and (b), respectively. It can be seen that the response to trams travelling across the switch in the two different directions differs in amplitude and frequency content. The correction of the geometry of PS2 in July 2011 reduced the peak strain measured for the crossing of trams bound for the Station by approximately $50 \%$, and increased the strain measured in response to Meadowhall-bound trams. The large stresses imposed on the stretcher bar by the misalignment of the switch resulted in the formation of a crack in the knuckle. As part of the remediation of the problem, prior to the realignment, the spring return was disconnected. Measurements undertaken in June 2011, with the cracked knuckle and disconnected spring return, show a change in the high frequency content of the signal for Station-bound trams, and a significant reduction in the strain experienced in response to the passage of a Meadowhall-bound tram. After realignment of PS2 there are apparent changes in the frequency content of both the Station- and Meadowhall-bound trams, likely to be a result of changes in the condition of the stretcher bar.

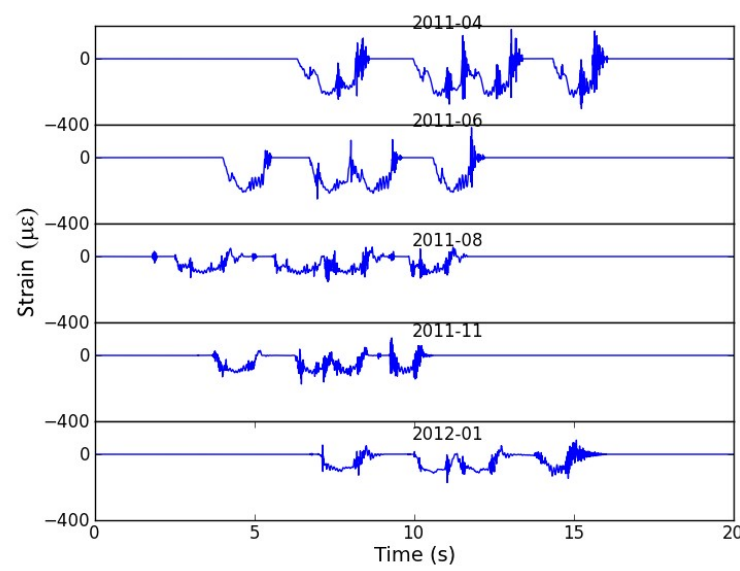

(a)

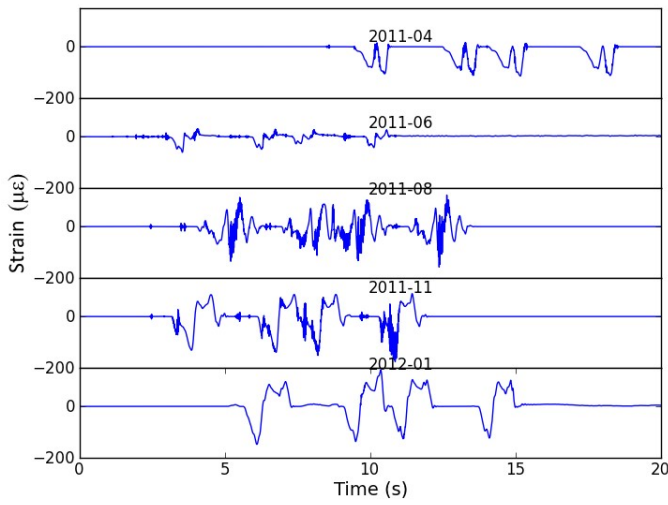

(b)

Figure 21. Strain measured by the sensor mounted on the knuckle of the stretcher bar induced by the passing of trams bound for (a) the Station and (b) Meadowhall. The directions are defined in figure 14. The dates indicate when the data were recorded

Figure 22 shows the strain signatures recorded by the FBG sensor mounted at the centre of the stretcher bar in response to Station- and Meadowhall-bound trams. The issues with the geometry of the switch in April and June 2011 caused large transient loading of the bar for Station-bound trams, which is significantly reduced after realignment. The disconnection of the spring return had an influence on the frequency content of the response in June 2011. There are also significant changes in the signature for 
Meadowhall-bound trams after switch realignment. While it is not possible currently to attribute these changes in signature to specific changes in condition, it is clear that there is a relationship between condition and the strain induced by the passing of a tram.

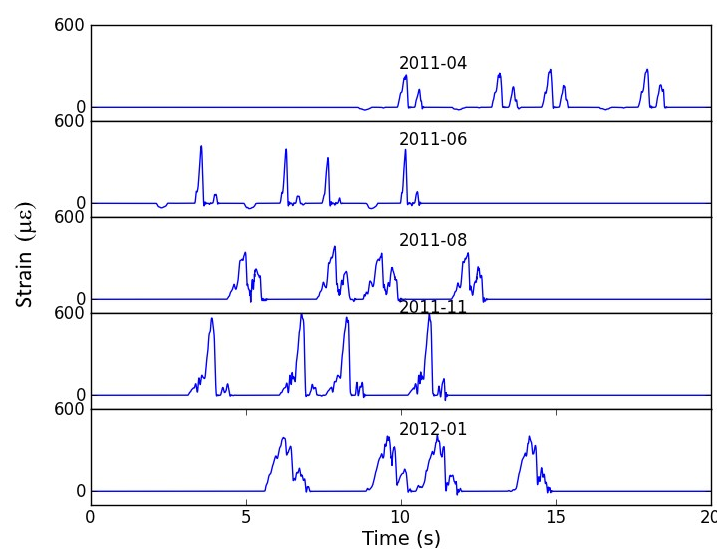

(a)

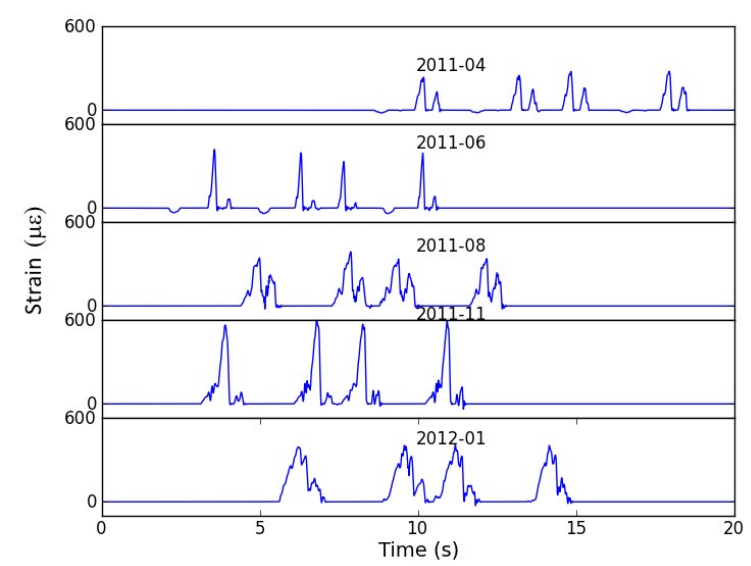

(b)

Figure 22. Strain measured by the sensor mounted at the centre of the stretcher bar induced by the passing of trams bound for (a) the Station and (b) Meadowhall. The directions are defined in figure 13.

\section{Summary}

Optical fibre Bragg grating sensors have been used to monitor changes in the dynamic strain signature induced in fishplates, stretcher bars and switchblades in response to the passage of a tram over the sensor location. The sensors were installed on railway track components under laboratory conditions and in-situ on a tram network. Visual inspection of the signals recorded indicates significant changes in the time series in response to changes in track condition, which may be indicative of changes in the conditions of the components. For the fishplates, a controlled series of experiments was undertaken in which the torque of the fishplate bolts was varied, both in a laboratory and on track. Both experiments indicated that it is possible to measure strain as an indicator of bolt-torque, and wavelet-based analysis of the time series facilitated the use of principal component analysis to classify the signals by bolt-torque. Further work is required on switchblades and stretcher bars, undertaking controlled experiments in which known changes in condition are imposed to allow the quantification of condition changes indicated by the strain signatures measured by the FBGs.

This work has demonstrated that optical fibre FBG sensor technology combined with sophisticated data analysis is able to reveal changes in the condition of safety critical rail components. The use of this technology would allow continuous monitoring of railway track components, offering significant safety and economic benefits over current practices.

\section{Acknowledgement}

This work was undertaken as part of the PMnIDEA project, funded by the European Commission's Seventh Framework Programme.

\section{References}

1 A.T.S. Worth and B Escartin-Claveria, Alstom's approach to railway condition monitoring, 6th IET Conference on Railway Condition Monitoring (RCM 2014), 1-6, 2014

2 V.J. Hodge, S. O'Keefe, M. Weeks and A. Moulds, Wireless Sensor Networks for Condition Monitoring in the Railway Industry: A Survey, IEEE Trans. Intell. Transp. Syst., 16, 1088-1106, 2015

3. S.J. Mihailov, Fiber Bragg Grating Sensors for Harsh Environments, Sensors, 12, 1898-1918 2012. 
4 T.K. Ho, S.Y. Liu, K.Y. Lee, Y.T. Ho, K.H. Ho, A. Mccusker, J.Kam, H.Y. Tam, S.L.Ho, An investigation of rail Condition monitoring by Fibre Bragg Grating sensors, Transactions Hong Kong Institution of Engineers, 16, 9-15, 2009. 5 L. Maurin, P. Ferdinand, G. Laffont, N. Roussel, J. Boussoir, S. Rougeault, High speed real-time contact measurements between a smart train pantograph with embedded Fibre Bragg Grating sensors and its overhead contact line, 6th International Workshop on Structural Health Monitoring, 1 \& 2, 1808-1815, 2007

6. R. M. Da Costa Marques Pimentel, M. C. Beirao Barbosa, N. M. S. Costa, D. R. F. Ribeiro, L. A. De Almeida Ferreira, F. M. M. Araujo, \& R. A. B. Calcada, Hybrid Fiber-Optic/Electrical Measurement System for Characterization of Railway Traffic and Its Effects on a Short Span Bridge, IEEE Sens. J., 8, 1243-1249, 2008

7. W. L. Li, N. Jiang, J. S. Liu, Y. Zhang, Train axle counters by Bragg and chirped grating techniques, Proc. SPIE, 7004, 70045J-1-4, 2008.

8. C.-L.Wei, C.-C. Lai, S.-Y.Liu, W.H.Chung, T.K.Ho, H.-Y.Tam, S.L.Ho, A.McCusker, J. Kam, K.Y.Lee, A fiber Bragg grating sensor system for train axle counting IEEE Sens. J., 10 art. no. 5482039, 2010

9 B. Allotta, P. D'Adamio, E. Meli and L. Pugi, Development of a new time domain-based algorithm for train detection and axle counting Vehicle System Dynamics 53, 1850-1875, 2015.

10 A. Laudati, G. Lanza, A. Cusano, A. Cutolo, G. Breglio, M. Giordano, A. Antonelli and G. Bocchetti, Railway Monitoring and Train Tracking by Fiber Bragg Grating Sensors: A Case Study in Italy, Proc. 4th European Workshop on Structural Health Monitoring, Poland, 183-189, 2008.

11 S.K.K. Hussaini, B. Indraratna, J.S. Vinod, Application of optical-fiber bragg grating sensors in monitoring the rail track deformations Geotechnical Testing Journal 38, 389-397, 2015

12 P. Boffi, G. Cattaneo, L. Amoriello, A. Barberis, G. Bucca, F. Bocciolone, A. Collina, M. Martinelli, Optical Fiber Sensors to Measure Collector Performance in the Pantograph-Catenary Interaction, IEEE Sens. J., 9, 635-640, 2009. 13 S.Li, Q.Ning, and H.Chen, Rail elevated bridge bearing displacement monitoring based on FBG sensor Applied Mechanics and Materials, 178-181, 2034-2037, 2012.

14 S. J. Buggy, S.W. James, S Staines, R. Carroll , P. Kitson, D. Farrington, L. Drewett , J Jaiswal and R.P. Tatam Toward track component condition monitoring using optical fibre Bragg grating sensors Proc. of SPIE Vol. 8421, $84213 \mathrm{M} 2012$

15. HSE 2003, Derailment at Southall East, Sunday 24 November 2002: HSE publishes investigation update derailment caused by a broken fishplate. http://www.hse.gov.uk/press/2003/e03027.htm, accessed 17th December 2012.

16 J.M. Lopez-Higuera, Handbook of Optical Fibre Sensing Technology, Wiley-Blackwell, 2002.

17. Y.J. Rao, In-fibre Bragg grating sensors, Meas. Sci. Technol., 8, 355-375, 1997

18. F. Mennella, A. Laudati, M. Esposito, A. Cusano, A. Cutolo, M. Giordano, S. Campopiano and G. Breglio. Railway monitoring and train tracking by fiber Bragg grating sensors, Proc. SPIE, 6619, H6193-H6193, 2007.

19. S. Kaewunruen and A. Remennikov, Dynamic effect on vibration signatures of cracks in railway prestressed concrete sleepers, Adv. Mat. Res., 41-42, 233-239, 2008.

20. W.Li, H. Li, J. Cheng, X. Huang, J. Pan, C. Zhou, M. Yang, Displacement monitoring of switch track and its slab on a bridge of high speed railway by FBG, Proc. SPIE . 7753, art. no. 775375, 2011.

21. W. Li, X. Huang, J. Cheng, J. Pan, Strain-temperature monitor of high speed railway switch by fiber Bragg grating gauges, Proc. SPIE ., 7656, art. no. 76565E, 2010.

22. H.Y. Tam, S.Y. Liu, B.O. Guan, W.H. Chung, T.H.T. Chan and L.K. Cheng, Utilization of fiber optic Bragg Grating sensing systems for health monitoring in railway applications, 6th International Workshop on Structural Health Monitoring, 1 \& 2, 1824-1831, 2007

23. C.C. Lai, J.C.P.Kam, D.C.C.Leung, T.K.Y.Lee, , A.Y.M.Tam , S.L.Ho, H.Y.Tam, M.S.Y.Liu, Development of a fiberoptic sensing system for train vibration and train weight measurements in Hong Kong, Journal of Sensors, 2012, art. no. 365165, 2012.

24. C.Wei, , Q.Xin, W.H.Chung, S.-Y.Liu, H.-Y.Tam, S.L.Ho, Real-time train wheel condition monitoring by fiber Bragg grating sensors International Journal of Distributed Sensor Networks, art. no. 409048, 2012,

25. D.R. Anderson, Detecting flat wheels with a fiber-optic sensor joint, Rail Conference on Restoring and Upgrading Rail Infrastructure, Rolling Stock and Systems, 31, 25-30, 2006.

26. D. Kang and W.Chung, Integrated monitoring scheme for a maglev guideway using multiplexed FBG sensor arrays, NDT and E International, 42, 260-266, 2009

27. K. Schröder, W. Ecke, M. Kautz, S. Willett, A. Tchertoriski, M. Jenzer, G.Kaluza, Fiber optical sensor network embedded in a current collector for defect monitoring on railway catenary, Proc. SPIE, 6585, U203-U210, 2007. 28. K. Schröder, W. Ecke, M. Kautz, S. Willett, M. Jenzer, and T. Bosselmann, An approach to continuous on-site monitoring of contact forces in current collectors by a fiber optic sensing system, Opt. Las. Eng., 51, 172-179, 2013. 
29. L. Maurin, P. Ferdinand, G. Laffont, N. Roussel, J. Boussoir, S. Rougeault, High speed real-time contact measurements between a smart train pantograph with embedded Fibre Bragg Grating sensors and its overhead contact line, 6th International Workshop on Structural Health Monitoring, 1 \& 2, 1808-1815, 2007

30 M.L. Filograno, P. Corredera Guillen, A. Rodriguez-Barrios, S. Martin-Lopez, M. Rodriguez-Plaza, A. Andres-Alguacil and M. Gonzalez-Herraez, Real-Time Monitoring of Railway Traffic Using Fiber Bragg Grating Sensors, Sens. J. 12, 8592, 2012

31. A. Barreda, T. Molina, E. Valero, S. Recuero, Use of fiber optic sensors for measurement railway vibrations, Proc. SPIE, 7653, 765345-1-4, 2010.

32. I. Bennion,; J.A.R Williams, and L Zhang, UV-written in-fibre Bragg gratings, Optical and Quantum Electronics 28, 93-135, 1996

33 P.J. Lemaire, , R.M. Atkins, V. Mizrahi, and W. A. Reed, High pressure H2 loadings as a technique for achieving ultrahigh UV photosensitivity and thermal sensitivity in GeO2 doped optical fibres, Electronics Letters 29, 1191-1193, 1993.

34 H. Patrick, S.L. Gilbert, A. Lidgard, M.D. Gallagher, Annealing of bragg gratings in hydrogen-loaded optical fiber, J. Appl. Phys. 78, 2940-2945 (1995).

35. http://www.pybytes.com/pywavelets/contents.html, last accessed 23rd December 2015.

36. I.T. Jolliffe, Principal Component Analysis, Series: Springer Series in Statistics, 2nd ed., Springer, NY, 2002,

37 C. Cortes, and V.N. Vapnik, Support-Vector Networks, Machine Learning, 20, 273-297 1995.

38. D. Albanese, R. Visintainer, S. Merler, S. Riccadonna, G. Jurman, C. Furlanello. mlpy: Machine Learning Python, 2012. arXiv:1202.6548, last accessed 23rd December 2015.

39 R. Yan, Base Wavelet Selection Criteria For Non-StationaryVibration Analysis In Bearing Health Diagnosis, PhD Thesis, University of Massachusetts, 2007.

40. http://wavelets.pybytes.com/wavelet/rbio3.1 
2016-03-22

Railway track component condition monitoring using optical fibre Bragg grating sensors

\section{Buggy, Stephen J.}

Buggy SJ, James SW, Staines S, et al., Railway track component condition monitoring using optical fibre Bragg grating sensors. Measurement Science and Technology, Volume 27, Issue 5, 2016, Article number 055201

https:doi.org/10.1088/0957-0233/27/5/055201

Downloaded from Cranfield Library Services E-Repository 